\title{
When Anisotropy of Vacuum Set the Space Isotropy
}

\author{
A. G. Syromyatnikov \\ Department of Physics, St. Petersburg University, Universitetskaya nab., St. Petersburg, Russia
}

Email address:

alsyromyatnikov@mail.ru

\section{To cite this article:}

A. G. Syromyatnikov. When Anisotropy of Vacuum Set the Space Isotropy. International Journal of High Energy Physics. Special Issue: Breaking of Space Symmetry in the Masses Spectrum Problem. Vol. 3, No. 1-1, 2016, pp. 9-14. doi: 10.11648/j.ijhep.s.2016030101.12

Received: June 19, 2015; Accepted: June 20, 2015; Published: March 14, 2016

\begin{abstract}
Results of some theoretical and experiment investigations of space-time anisotropy of Galactic gamma-rays angular distribution within the frame of the space rays generation mechanism on a method of direct transformation of intergalactic gamma-rays to the current on spin shock-waves are presented. However, the source anisotropy origin is unknown. Here I show that on Fermi telescope data brightness at the edge of the area, limited $20^{\circ}$ Galactic longitude, twice higher the brightness of diffuse radiation. Thus the range of the observed distribution mapping of gamma rays from the Galactic longitude of theoretical (intensity of above-average level) in fact is limited above $20^{\circ}$ strict accordance with the theory. I found that the Galactic gamma - ray angular anisotropy axis has following coordinates: longitude $1=96^{\circ}$, latitude $\approx 20^{\circ}$, that corresponds in the second equatorial coordinate system: right ascension $\alpha=271^{\circ}$, declination $\delta \approx 40$ - supernova residuals Cygnus X-3 on periphery.
\end{abstract}

Keywords: Galaxy Gamma-Rays, Spin Shock-Waves, Eight Spin Shock Waves Model, Fermi Space Telescope

\section{Introduction}

In the Galactic Gamma-Astronomy the gamma-radiation is produced by cosmic rays. Cosmic rays at $90 \%$ composed of electrically charged protons and carry an electric current. Galactic Gamma-radiation is characterized by sharp anisotropy longitude distribution (energy more than $70 \mathrm{MeV}$ ) in the Galactic latitudes $\pm 10^{\circ}$ [1] (see Fig. 1). Sources can be and $\mathrm{x}$-ray bursters [2] - flaring which can last from several minutes up to several tens of hours. Flash development time $0.1-5 \mathrm{~s}$, decay 3-100 s. The majority of bursters within $30^{\circ}$ from the direction of the Galactic Center, indicating their affiliation with spherical Galaxy subsystem. Absolute luminosity x-ray bursters during a flash of $10^{37}-10^{38} \mathrm{erg} / \mathrm{s}$, the total energy of the radiation during this time in the $\mathrm{x}$-ray range $10^{38}-10^{39} \mathrm{erg}$. Between flashes bursters are slowly changing $\mathrm{x}$-ray sources of two orders of magnitude less luminosity and energy of photons is less than $15 \mathrm{keV}$.

The radius of the radiating area is estimated at $10 \mathrm{~km}$. Where observed flashes as x-ray and optical radiation for three bursters (with delays 3 s). It is accepted that bursters are a close binary system of red dwarf and neutron stars. X-ray emission from the barster in a calm phase caused by release of gravitational energy accretion substances. On the theory [2] at the time of ignition of hydrogen or helium accretion layer develops thermal (helium) flash, resulting in a cascade of output power for a short time in the form of x-rays. In addition, found a few gamma-bursters, short-term sources of gamma radiation in the constellation Aquila, etc.

In [1] the mechanisms of generation of cosmic rays by way of direct conversion of extra-Galactic gamma-ray (as well as radio-frequency masers and x-ray bursters) into electric current to spin shock waves in a neutral interstellar medium etc. The task was the following statement:

Generate currents in the ground experiment of focusing radio waves to dielectric target (see link in [1]) is in the range of angles from $32^{\circ}$ to $104^{\circ}$ from the axis of anisotropy in the direction towards incident radiation. The currents passed through the annular surface along the axis (the axis of anisotropy) source of the incident radiation flux. Reverse currents, extending in the direction of incident radiation, are limited to the limit angle $104^{\circ}$. Theory allows determining the distribution of currents in the corners, measured from the axis of anisotropy. Generate currents in the Eight spin shock waves model (see, e.g., [1] and [3]) in the inner configuration space is in the same range of angles from $32^{\circ}$ to $104^{\circ}$ from the axis of anisotropy. When you set the size of the target on the theory is defined a full of electric charge 0.01 Coulomb, characteristic of these processes. Observation of the Galactic radiation has the ability to measure its intensity only per unit area, rather than across the entire annular surface. However, based on the symmetry of the distribution of currents along the annular 
surface that takes place in a land lab experiment, in [1] it was performed and for the Galactic radiation. Then the comparison between the theory value of current through the annular surface based on the Galactic longitude and intensity of radiation per unit of that surface becomes more defined. To understand the tasks in this formulation was subjected to a theoretical treatment of the pulse of a discrete source of x-ray radiation of barster depending on the time. During pulse according to the theoretical model the vector of the current runs through the same range of angles in the inner configuration space that and in range of Galactic longitudes. In Fig. 2 [1] built the pulse for x-ray burster MXB 1730-335 usually type 1 (write the Flash made equipment the artificial satellite of the Earth "Astron", 1983 [2]) compared with the pulse current basic model eight spin shock waves, delayed from the background ( 28 counts in $0.83 \mathrm{sec}$.). Burster is far from the Galactic plane. Therefore, absorption in the structure of the Galaxies can be neglected. There was a precise agreement between generated currents (theory) with the burster radiation, characterized by the standard deviation of \pm $1 \%$ in intensity in relative units within the sensitivity of the equipment. Thus, it was found that the spin angular momentum conservation law (equation of dynamics of spin shock waves) in the x-ray range is fulfilled exactly in real time. On the other hand according to the Noether's theorem conserving of spin angular momentum means the isotropy of space and time.

Further, despite the fact that the source of the incident radiation is not known in advance, the axis of anisotropy can be defined, but only at the end of the match the angular distribution of the observed intensity and theory.

Following results were obtained: the axis of anisotropy on Fig. 1 (bottom) with satisfactory agreement between distributions of gamma-radiation from the Galactic Center and the spiral arms on the one hand, and currents on the other, determined by the Galactic longitude of $276^{\circ}\left(-84^{\circ}\right)$. It is close to the most powerful source of the Galactic source Sail-X (this nebula with pulsars, formed after the explosion of a supernova).

On the other hand falls (extra-Galactic) radiation should be directed towards Galactic rays in the opposite direction: $1=$ $-84^{\circ}+180^{\circ}=96^{\circ}$. Although some distance and a powerful source of the Swan X-3 in $12 \mathrm{kpc}$ from the Sun, but in the immediate surroundings of the notable individual sources of gamma-rays not. Apparently, it was out of there in our Galaxy are the original gamma rays transforming into electrically charged cosmic rays. The axis of anisotropy has following Galactic coordinates: longitude (exactly) $1=96^{\circ}$, latitude $\approx$ $20^{\circ}$, that corresponds in the second equatorial coordinate system: right ascension $\alpha=271^{\circ}$, declination $\delta \approx 40^{\circ}$. Thus was received experimental confirmation in these processes in a range of Galactic longitude $\left(-84^{\circ},+20^{\circ}\right)$ the fairness of the law of conservation of spin angular momentum of electromagnetic field as part of the total angular momentum. This is due to the fact that in the spin-flip processes on a front spin shock wave integral conservation of the spin fulfilled by way of expanding the class of solutions in generalized functions of jumps.

A special place in the task output gamma-quanta is the area of Galactic longitude $20^{\circ}-40^{\circ}$, where is clearly consistent failure to restrict the data stripe widths $\pm 10^{\circ}$, and a record of sources up to $30^{\circ}$ Galactic latitude, as well as to be considered in the light of the possible effects of quantum gravity with a nonzero torsion.

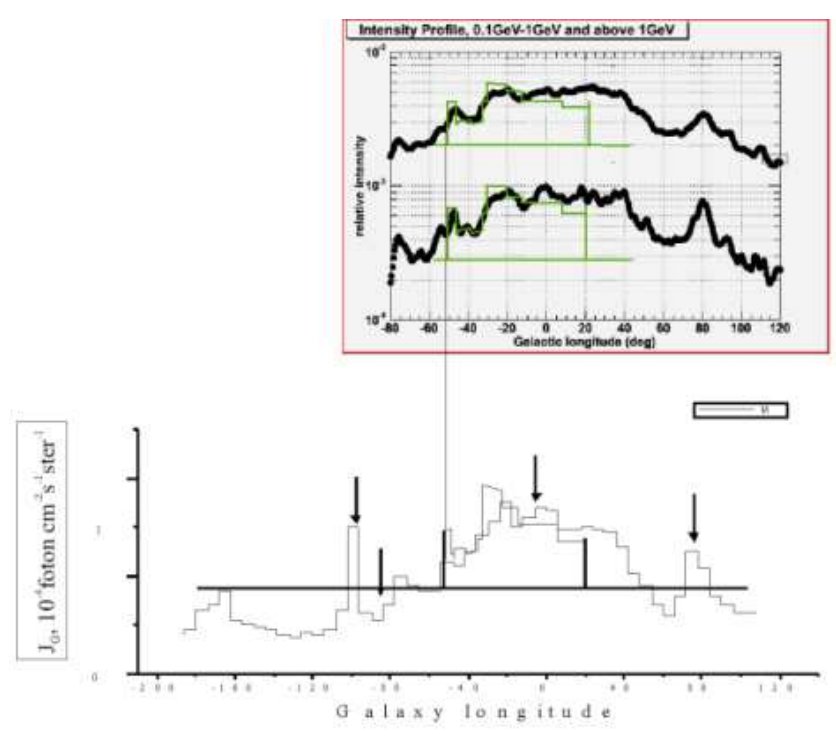

Fig. 1. Longitude distribution of Galactic Gamma-radiation [2] $J_{G}$ (energy more than $70 \mathrm{Mev}$ ) in the latitudes range $\pm 10^{\circ}$ (line across the range of longitudes) in relation to the distribution of Galactic currents on basic Eight spin shock waves model built (thin line ascending from the upper ends of two fatty vertical line segments beginning with the horizontal line middle level) from the average level of gamma radiation in comparison with data [4].

The radiation from higher latitudes (see Fig. 2) in the center of the Galaxy next $20^{\circ}$ (extreme value theory), the diffuse radiation is reduced to the amplitude of the average level. At the top there is a clear peak near longitude $-50^{\circ}$ in accordance with the theory. Its position is indicated by a vertical line.

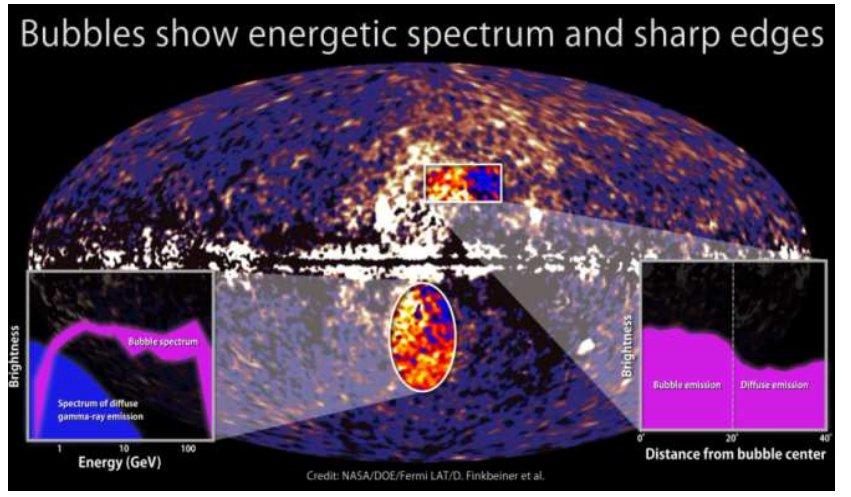

Fig. 2. Picture [4] gamma-radiation of two spheres (bubbles) with a diameter of 25000 light years with touch point at the center of the Galaxy.

In the corner to the right brightness at the edge of the sphere, limited $20^{\circ}$ Galactic longitude, twice higher the brightness of diffuse radiation (its intensity here is below average level). In the corner of the left is the spectrum of gamma-radiation bubble characterized by absorption of about $600 \mathrm{GeV}$ $(0.6 \mathrm{TeV})$ 
In this work, all these issues are considered (according to [6]) in the setting of mapping theory of Eight spin shock waves model profile intensity of Galactic diffuse gamma radiation and gamma radiation from bubbles in the center of the Galaxy, according to a large gamma-ray space telescope Fermi (GLAST) [4]. The area also included $\pm 10^{\circ}$ Galactic latitude of Fig. 1, an additional gamma rays of two spheres with a diameter of 25000 light years (30000 light years on neutrinos telescope data (see [6])) with touch point at the center of the Galaxy (see Fig. 2). In the corner to the right brightness at the edge of the area, limited $20^{\circ}$ Galactic longitude, twice higher the brightness of diffuse radiation (its intensity here near and below average level). This demonstrates that the range of the observed distribution mapping of gamma rays from the Galactic longitude of theoretical (intensity of above-average level) in fact is limited above $20^{\circ}\left(104^{\circ}\right.$ Eight spin shock waves model) in strict accordance with the theory.

In theory, both spheres can be viewed as a quantum-field vacuum condensate of gravitating spinors [5] of helium-3 for which give the value of diameter of condensate theory -30000 light years.

In the corner of the left is the spectrum of gamma-radiation sphere characterized by absorption of about $600 \mathrm{GeV}(0.6$ $\mathrm{TeV}$ ). In theory [5], the absorption can corresponds the threshold of gravitational mass and inertial mass differences $\left|\mathrm{M}_{\text {in }}-\mathrm{M}_{\text {grav }}\right|<10^{-12}$ particles scale of GUT $10^{15} \mathrm{M}_{\mathrm{p}}$.

In all cases above $20^{\circ}$ Galactic longitude intensity of diffuse gamma radiation quickly subsides, so that the theory of Eight spin model profile compared with the intensity of Galactic diffuse gamma radiation from large gamma-ray space telescope (GLAST) is a experimental distribution of diffuse Galactic Gamma-ray bursts very accurately, in all range of the Galactic longitude within the accuracy of measurements in the presence of extra-galactic sources, Sails, etc.

All of this confirms the conservation low of the spin angular momentum, as part of a full and the isotropy of space-time across the Galaxy.

\section{Extra-Galactic Sources of Gamma Radiation}

Let's look at the brightest gamma-ray sources (website: Wikipedia)

Table 1. Extra-galactic objects closest to the direction $l=96^{\circ}$ of the Galactic axis of anisotropy.

\begin{tabular}{|c|c|c|c|c|c|c|}
\hline No & $A^{1}$ & $\mathbf{B}^{2}$ & $\mathrm{C}^{3}$ & $\mathrm{D}^{4}$ & $\mathbf{E}^{5}$ & $F^{6}$ \\
\hline 1 & M74 NGC 628 & 24.175 & 15.8 & 106.59 & -45.3 & 30 \\
\hline 2 & M15 NGC 7078 & 21.5 & 12.2 & 109.3 & -49.4 & \\
\hline 3 & NGC 1313 & 49.5 & 66.5 & 108.9 & 8.1 & \\
\hline 4 & NGC 4151 & 182.4 & 39.5 & 91.43 & 75.40 & 60 \\
\hline 1 & Galactic axis of & 200.9 & 40.1 & 96 & 75.4 & \\
\hline 2 & anisotropy & 210 & 13.8 & 96 & -45.3 & \\
\hline 3 & & 250 & 58 & 96 & 8.1 & \\
\hline \multirow[t]{3}{*}{4} & & 271 & 64 & 96 & 20 & \\
\hline & Galactic periphery & & & & & \\
\hline & Cygnus X-3 & 308.125 & 40.57 & 166.115 & 0.286 & 0.03 \\
\hline
\end{tabular}

${ }^{1}$ Extra-galactic object

${ }^{2}$ the right ascension $\alpha$, degrees

${ }^{3}$ Declination $\delta$, degrees

${ }^{4}$ Galactic longitude 1, degrees

${ }^{5}$ Galactic Latitude b, degrees

${ }^{6}$ The distance to the Earth, million light years

This table lists the coordinates of extra-galactic gamma ray sources in the second equatorial coordinate system by catalogue in the Galactic coordinate system. For each source were converted to the coordinates of the Galactic axis of anisotropy on the latitude of the corresponding source. For example, the galaxy NGC 4151 has Galactic longitude $91^{\circ}$, longitude $96^{\circ}$ closest to the Galactic axis anisotropy (AGA).

The definition of the Galactic latitude $75.4^{\circ}$ to latitude AGA gives a very close match between AGA and NGC 4151 on decline $40.1^{\circ}$ and $39.5^{\circ}$ respectively, with little difference between $19^{\circ}$ in right ascension. In all other cases, the decline of the galaxies, M74 M15, NGC 1313 and AGA (Galaxy) are also close, but direct ascent AGA and galaxies are opposite in direction. Thus, in relation to individual gamma sources coordinates $\alpha=201^{\circ}, \delta=40^{\circ}$ Galactic axis of anisotropy is the location of the high-latitudinal $b=75.4^{\circ}$ galaxy NGC 4151. In this direction (towards the NGC 4151) in experiments [7] chart registered local maximum output power. Same output energy maximums against match directions of anisotropy by right ascension axis $210^{\circ}, 250^{\circ}$ and in relation to all other sources the M74 to 30 million light years, M15, NGC 1313. In Fig. 3, all these directions are indicated by their numbers on a chart of average annual release of seismic energy on the number of the strongest earthquakes occurred in the period from 1990 to 2015 timeframe (according to the web-site: ceme.gsras.ru). 
The number of earhouakes over the $1990-2015$ years

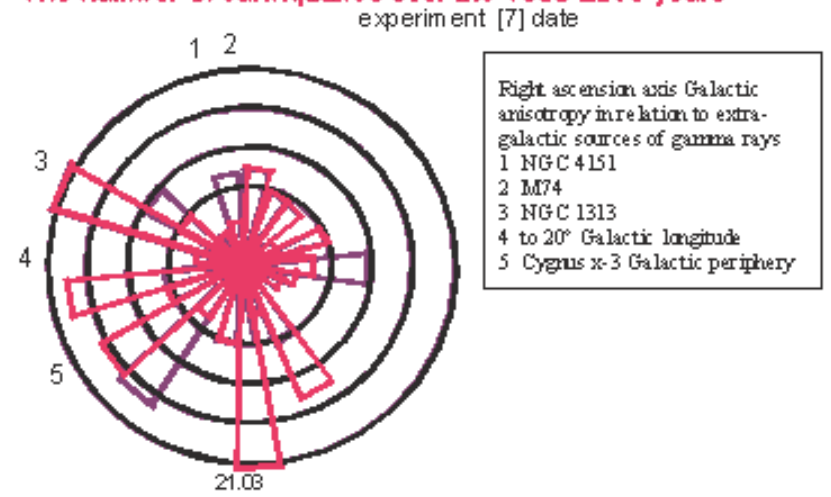

Fig. 3. The direction of the Galactic axis of anisotropy in relation to extragalactic gamma ray sources in the second equatorial coordinate system built on a chart of average annual release of seismic energy on the number of the strongest earthquakes occurred in the period from 1990 to 2015 timeframe (red). There are also dark blue postponed parts of maximum yield energy diagram [7] at their intersection with the $14^{\circ}$ sectors earthquake chart.

The array of source data was averaging method of sliding window by 5 points. After that was the background subtraction. Then the resulting data was encapsulated within $14^{\circ}$ sectors. Without these operations with source data diagram anisotropy is blurred.

The correlation coefficient between the number 201, 210, 250, 271, 308 of the Galactic axis of anisotropy and the number 190, 190, 250, 250, 330 directions of energy output in the chart maximums [7] reaches 0.995 . The standard deviation between the areas on the border $10^{\circ}$ sector of graph [7] $2^{\circ} \pm 9^{\circ}$ does not extend beyond one sector.

As a result of the mapping of Galactic axis of anisotropy and extragalactic sources identified two directions: the first $\alpha$ $=271^{\circ}$, which has no correlation with any extragalactic gamma radiation sources, and $\alpha=201^{\circ}, \delta=40^{\circ}$-acting within the limits of the allowable spread across the first-due to the gamma radiation the brightest galaxy NGC 4151. This Galaxy is far from Earth in a distance 60 million light years. Our galaxy with a diameter of 100 thousand light years from this distance is visible at an angle $0.1^{\circ}$. In our Galaxy to this list, you can attach a powerful source of Swan X-3 on the Galactic periphery due to its unusual properties. In particular, a large output of muons with energy of $1 \mathrm{TeV}$ in one act of interaction, at least 20 times more than it should have been under the assumption that they are born TeV's photons from a Swan X-3, [8] and a fairly large angular dispersion of $7^{\circ}$ their pulses around the direction of the source. The angular variation is explained in [8] scattering in the atmosphere. In [8] it is believed that from the source come other particles of ultrahigh energies other than photons. It is believed that these particles should be neutral and have zero mass or a small $(\mathrm{GeV})$. On the other hand, now this kind of abnormal output of muons created in experiments on tevatron. What follows from this it is discussing below.

\section{Discussion and Conclusions}

1. Data distribution of a galactic longitude of gamma radiation does not allow to determine the latitude of the Galactic axis anisotropy (AGA). Fixing the Galactic latitude $\mathrm{AGA}$ on $20^{\circ}$, at the Centre of the sphere, when the manifestation of anisotropy is a theoretical limit, leading to the identification of directions of AGA with data [9-10] for the measurement of cosmological vector AG. In this regard any extragalactic sources were revealed.

2. Fixing the Galactic latitude AGA on the longitude $75^{\circ}, 4$ of the galaxy NGC 4151 meets all the requirements to the source. The Galactic axis of anisotropy is aimed across the direction of AG. The distance to the "eye of Sauron"- galaxy NGC 4151 (http://www.gismeteo.ru) is 19 Megaparsec (around 60 million light years).

3. According to [8] Cygnus X-3 is the only Galactic source, recognized as a natural Accelerator, producing beams of particles with energy up to $10^{16} \mathrm{eV}$. In scatterings with slow protons, which necessarily are present in that object and its surroundings, the energy in the system's Center of mass, as it is known, is up to $4 \mathrm{TeV}$. That's enough to approach the threshold on the difference between gravitational and inertial masses less than $10^{-12}$ scale of $10^{15}$ mass of a proton.

In fig. 2 on the left you can see attenuation spectrum is the energy of $1 \mathrm{TeV}$. In the muon anomaly registered significant effect of muon jets outside the accelerator tract that in theory is discussed in [12] in the oscillator model string space-time torsion. This increases the opportunity to consider this source as a source of galactic cosmic rays.

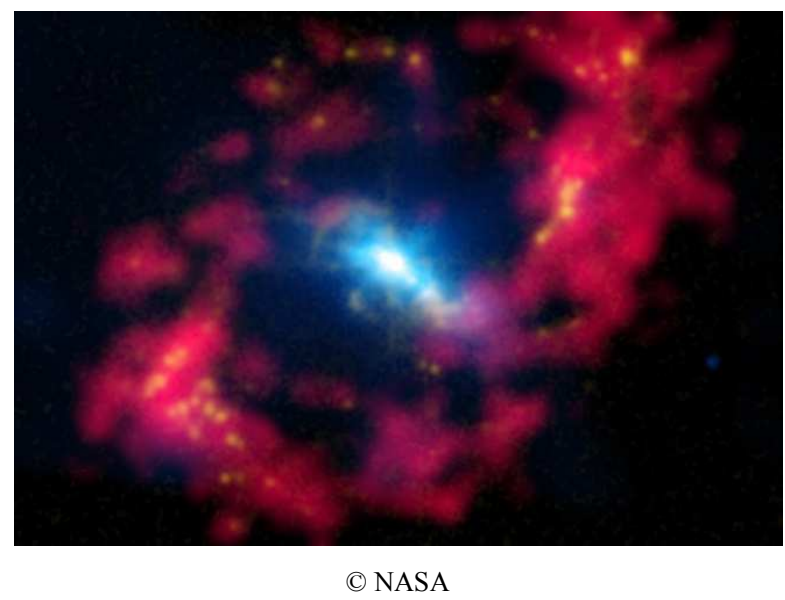

Fig. 4. NGC 4151 is a Galaxy in the constellation Canes Venatici, it is visible from the Earth and is readily available for observation by amateur astronomers.

The Galaxy won its name for similarity to the object from the novel J. In J. R. R. Tolkien's the Lord of the rings (http://www.gismeteo.ru). "To get a complete picture of the astronomers combined data from three independent research centers. Yellow belongs to the spectrum of ionized hydrogen. This image was obtained with a telescope JKT, which is located on the Canary Islands. Blue color-image in the X-ray region, observed by Chandra space telescope. Using a set of radio telescopes of the VLT registered red spectrum (color?) neutral hydrogen» www.cosmonews.ru).

1. Thus, in cases, the angular distribution anisotropy-Galactic gamma-rays and pulsars using Eight 
spin shock waves is determined by the paradoxical way, so this sets the isotropy of space-time in according to the Noether's theorem: conservation of spin angular momentum, as Spin dynamics low for the shock waves, means the isotropy of space - time. Actually Maxwell equations for a dust-like medium of charged particles in the flat space

$$
\partial^{\alpha} \partial_{[\alpha} A_{\mu]}=\frac{4 \pi}{c} \pi \rho_{e} u_{\mu}, u_{\mu}=\frac{e}{c} A_{\mu}+\partial_{\mu} \sigma
$$

in the Lorentz gauge

$$
\partial^{\alpha} \partial_{[\alpha} A_{\mu]}=\frac{c}{e} \partial^{\alpha} \partial_{[\alpha} u_{\mu]}=\partial^{\alpha} \partial_{\alpha} A_{\mu},
$$

reduced identically to the conservation law of density tensor spin $\mathrm{S}_{\mu \nu}^{\alpha}$ of the self-consistent electromagnetic field $\mathrm{A}_{\mu}$

$$
\partial_{\alpha} S^{\alpha}{ }_{\nu \mu} \equiv \partial^{\alpha}\left(u_{[v} \partial_{\alpha} u_{\mu]}\right)=0,
$$

$\rho_{\mathrm{e}}-$ an electric charge density, $\mathrm{u}_{\mu}-4$-speed of particles $\left(\mathrm{u}^{2}=\right.$ $1), \mathrm{e}-$ an electron charge, $\partial_{\mu}$ - partial derivative, $\mathrm{c}-$ the speed of light in vacuum, $\sigma(\mathbf{x}, \mathrm{t})$ - the gauge function.

Eight spin shock waves model is based on an accurate solution of the Maxwell equations for a dust-like medium of charged particles. As a result, it was found that the Lorentz boost and Euler's angles are functions of the variable which satisfies the linear wave equation. In particular

$$
\begin{aligned}
& u^{0}=\gamma=\gamma_{0} \operatorname{ch} \varphi, \\
& u^{1}=\gamma^{0} \operatorname{sh} \varphi, \\
& \Delta \varphi=0 .
\end{aligned}
$$

For small angles, the "eight spin" model is similar to the fundamental $\mathrm{O}(3)$ model.

On the other hand according to [11] in the system of gravitating fermions in flat space, taking into account the producing of the masses, there is a quantum effect of spontaneous breaking of symmetry of the vacuum as in magnetism. The resulting selection area can be mapped to the direction of the cosmological vector $\mathrm{A}_{\mathrm{G}}$.

The mechanism of action of new forces [9-10], as in very simplified form of proportional to the vector-potential of electromagnetic field of constant tension proportional to coordinate, you can consider the analogy with quantum oscillator model [6] to string [5] used to explain the effects of the muon anomaly in hadronic collider [6]. Despite the smallness of the torsion constant of effective interaction between the fermions, the constant through the exchange of torsion pseudovector by the smallness of its mass was more than a hundred times stronger than the electromagnetic fine structure constant. The parameters of the model are calculated from the conditions for compensation of the infinite vacuum Hartry-Fock's energy shift of the main level energy by the energy of torsion strings in physical 4-dimensional space-time. The gravitational effect of Conformal gauge theory of gravitation for tensor field mediated by the two rank tensor potential of torsion was identical to the action of the B-string, with explicit by replacing the variables is given to mean continual integral on fields. With these settings, the dynamic equations of motion of the strings lead to the displacement of the center of mass at 1-2 cm across the tract of the collider for its borders (the gravitational effect does not affect the local dynamics of interacting fields, but only leads to a shift of the center of masses), and displaced masses in experiments [9-10] In the latter case, the relaxation time of 1 minute masses, as it is observed.

Qualitatively, this approach has all the basic features of a hypothetical interactions in the theory [10]-the energy of the strings is proportional to the energy of the quantum field vacuum, and according to $[5,12]$ spin tensor density gauge field in vacuum is limited (by the density of helicity). Since the tensor density spin of gauge electromagnetic field is proportional to the vector-potential, this puts the cumulative limit. Quantum electrodynamics rather then takes the compact view.

\section{Conclusion}

Many shared the view that, in determining the spectrum of masses should be taken of the gravity. All this investigation can be directly generalized for the case of the space strings in terms of the cone space topology which has no difference from the plane space - time everywhere except for a small angle, which is proportional to the mass density of the string by Nilsen - Olsen, which is similar to the mass angle of leptons anisotropy in the author's Conformal Gauge Theory of Gravitation (1996). Theoretically, the birth of the masses on Higgs's mechanism of the Standard Model, taking into account the gravity inevitably accompanied by anisotropy of vacuum that whatever was said about the geometry of space-time. On the other hand the globality of spatial anisotropy is due to the conformal symmetry.

\section{References}

[1] SYROMYATNIKOV A. G., Vestnik Sanct Peterburgskogo universiteta. Ser. 4. Vip. 2 (2012) 108-112; SYROMYATNIKOV A. G., Vestnik Sanct Peterburgskogo universiteta. Ser. 4. Vol. 2 (60) Vip. 1 (2015) 88-92.

[2] Gamma-ray astronomy, Physical encyclopedia/HL. Ed. Prokhorov A. M., (Sov. Encyclopedia, Moscow, T1. Aaron-Bohm effect-long lines, 1988): web-site: http://en.wikipedia.org/wiki/Gamma-ray_burst.

[3] PROSHIN Yu. N., USEYNOV N. H., Magnetic break with spin rotation, UFN 165 No. 1 (1985) 41-87.

[4] Website (2015): glast.gsfc.nasa.gov: Diffuse Hard X-Ray/Gamma-Ray Emission from Galaxy and local group of galaxies Galactic Diffuse Emission: MIZUNOI T., TAKAHASHI T., TAKAHASHI H., KATAGIRI H., FUZUKAWA Y. and OHSUGI T. (Hiroshima Univ.); STANLEY D. Hunter, galactic diffuse gamma-ray emission, the EGRET Model, and GLAST, Science, July 23, 2004. NASA /GSFC Code 66/. 
[5] SYROMYATNIKOV A. G., Physical effects in Conformal Gauge Theory of Gravitation (LAP Lambert Academic Publishing GmbH \& Co. KG, Saarbrucken, Germany, 2012).

[6] SYROMYATNIKOV A. G., On some feature of possible torsion effects on observables at hadron colliders, Int. J. Geom. Meth. Mod. Phys (2015) (to be published) DOI: $10.1142 / \mathrm{S} 0219887815500802$.

[7] BAUROV Yu. A. et al., Phys. Lett. A. 311 (2003) 512.

[8] ARBUSOV B. A., JETP Lett. 42 Vip. 10 (1985) 430-432.

[9] BAUROV Yu. A., The anisotropy of cosmic rays and the global anisotropy of physical space, J. Mod. Phys. 3 (2012) 1744 .
[10] BAUROV Yu. A., On the structure of physical vacuum and a new interaction in Nature (Theory, Experiment and Applications) (Nova Science, NY, 2000).

[11] SYROMYATNIKOV A. G., Vestnik Sanct Peterburgskogo universiteta. Ser. 4. Vip. 2 (2009) 410-425.

[12] SYROMYATNIKOV A. G., Sovremenny nauchny vestnik. Ser.: Physics. No4 (100) (2011) 33-44. (DOI: 10.17686/sced_rusnauka_2011-236).

[13] TEILOR J. H., MANCHESTER R. N. and LYNE A. G., Catalog of 558 Pulsars, Ap. J. Suppl. Series 88 (1993) 529-568.

[14] Supernova Catalogue (Moscow State University, Astronomy institute, 2015). 